\title{
Using Protein Microarrays to Study Cancer
}

\author{
Arun Sreekumar and Arul M. Chinnaiyan \\ Departments of Pathology and Urology, Comprehensive Cancer Center, \\ University of Michigan Medical School, Ann Arbor, MI, USA
}

BioTechniques 33:S46-S53 (December 2002)

\section{ABSTRACT}

Microarrays or "biochips" provide an efficient way to achieve a global molecular perspective of human disease. DNA microarrays have been used extensively to generate transcriptional fingerprints of cancer. More recently, high-throughput techniques to study proteins have also been developed. Biochip-based microarrays containing spotted antigens or antibodies offer the ability to study protein-protein interactions, identify biomarkers, and study the humoral response to cancer. This review will focus on developments in the area of antigen and antibody microarrays and describe some of their applications to cancer research.

\section{INTRODUCTION}

Cancer is one of the leading causes of death in the Western world. Approximately one out of three individuals is affected by this disease, which is characterized by deregulated cell proliferation and cell death (33). The term cancer is used to describe cells that lose their ability to regulate growth, become immortal, invade surrounding tissue, and, in some cases migrate from their primary site of origin. A number of genetic, epigenetic, and environmental factors contribute to the development and progression of cancer, and thus, it will be advantageous to study these molecular regulations with a global perspective in mind.

Microarray or "biochip" technologies have the potential of revolutionizing the way we analyze and examine human cancer. By simultaneously gauging the expression of thousands of genes or proteins in clinical specimens, a wealth of data points are generated that coalesce to form a molecular fingerprint of a disease process. Tumor markers are proteins or substances that correlate with malignancy and may represent alterations from a normal state to a malignant process (35). These can be detected in solid tumors, lymph nodes, bone marrow, stool, ascites, and most conveniently, in serum or urine. Detecting or monitoring the levels of tumor markers may aid in diagnosis, staging, population screening, assessing response to treatment, and identifying occult metastatic or recurrent disease. The absolute presence of a tumor marker, or the relative level of the marker, distinguishes normal from malignant states. Several therapeutic strategies have targeted tumor markers, one example being Herceptin ${ }^{\circledR}$, a humanized monoclonal antibody directed against HER-2/neu, which is a transmembrane glycoprotein overexpressed in breast cancer, as well as other carcinomas. Protein microarray technologies will have a major impact on the discovery, characterization, and clinical application of cancer biomarkers.

As a result of the Human Genome Project, large collections of genes (and their encoded proteins) will be available for parallel functional genomic studies. Examples of these high-throughput technologies include high-density DNA, protein, and tissue microarrays. DNA microarray techniques have matured the furthest and are currently dominated by two basic platforms, oligonucleotide arrays and spotted cDNA arrays. These DNA biochips have been used to identify single nucleotide polymorphisms (21), genotype viruses (12), characterize signaling pathways elicited by various biological stimuli (16), define prognostic markers for cancer $(1,4,6,36,37)$, and classify tumors and predict clinical outcome $(3,4)$.

Establishing parallel analysis of protein function and quantity using biochips has been much more elusive, but recent studies by several groups have made significant advancements. Unlike hybridization, which involves interaction of linear DNA or RNA sequences, protein-protein interactions depend on folded 3-dimensional (3D) amino acid sequences. Maintaining the folded properties of proteins may pose a potential problem in the fabrication of protein microarrays. Furthermore, relative to hybridization reactions, folded proteins have greater sequence dependence and are prone to nonspecific interactions.

Conventional methods for detecting protein-protein interactions include the yeast 2-hybrid system, co-immunoprecipitation assays, and phage display. Similarly, quantitation of proteins has relied upon techniques such as 1D and 2D gel electrophoresis, immunoblotting, enzyme-linked immunosorbent assays (ELISAs), and radioimmunoassays (RIAs). Most of these wellestablished methods for protein characterization in cell and tissue extracts are not amenable to high-throughput applications and would benefit by implementation of a biochip format. For example, conventional 2D electrophoresis can readily resolve about 1000 proteins, which is only a small fraction of the total proteins expressed by a cell (26). Also, the requirement of large sample volumes for most of these assays makes them unattractive for analysis of precious samples, such as population-based serum 
collections. Furthermore, the development of high-throughput techniques to profile protein levels would help complement rapidly accumulating gene expression data.

Recent advances in high-throughput screening have led to the development of numerous biochip-based assay systems for monitoring protein expression and/or activities in cells and tissues. Most of these technologies rely on spotting either the antigen or antibody as baits on derivatized glass surfaces using high precision robotic arrayers. These chips are then probed with fluorescently labeled probes containing purified proteins, antibodies, cell lysates, or sera. This offers the potential of comprehensive proteomic analysis with very low sample amounts, at relatively low cost, and requiring less time. This review will focus on the major developments in microarray-based proteomics as it relates to cancer biology. To illustrate some of the techniques, we will provide examples from our own experience.

\section{ANTIGEN MICROARRAYS}

Arrays of antigens or proteins have many potential uses in cancer research and include the study of protein-protein interactions and humoral response to a tumor. The basic concept underlying the fabrication of antigen arrays is shown in Figure 1 and involves arraying purified proteins or complex biological mixtures onto modified solid matrices. The antigen microarrays are then incubated with a mixture of a reference sample (e.g., control sera) and test samples (e.g., cancer sera), each labeled with a spectrally resolvable fluorescent dye. The hybridized slides are analyzed, and the abundance of protein-antibody in the test sample relative to that of the reference sample is assayed. The simplest scenario involves an antigen array probed with

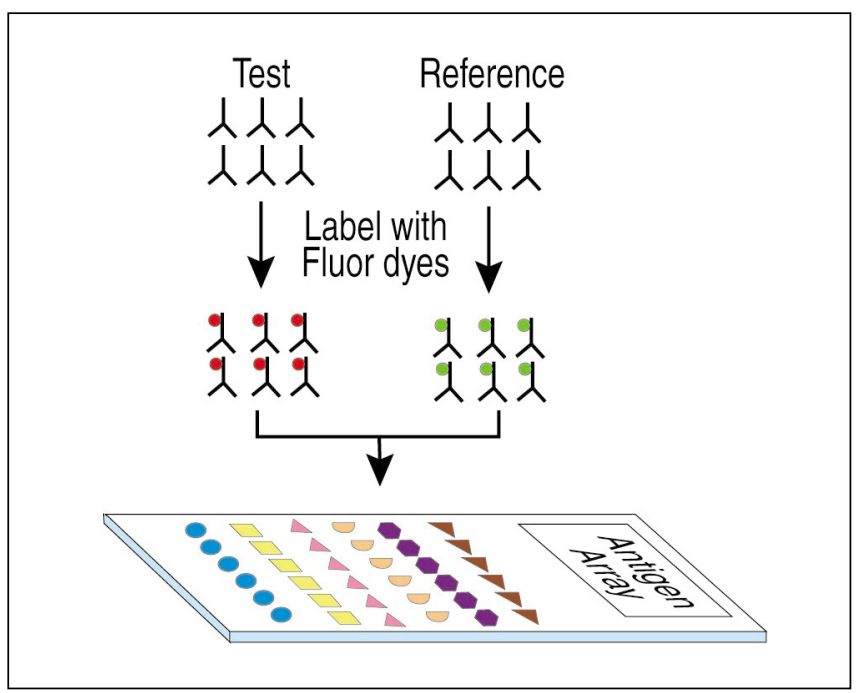

Figure 1. Schematic representation of an antigen array used to monitor protein-protein interactions and humoral response in cancer. Recombinant proteins or complex mixtures are arrayed onto modified solid matrices using a highspeed robot. The slides containing the microarrays are then incubated with a mixture of a reference sample (e.g., control sera) and test samples (e.g., cancer sera), each labeled with a spectrally resolvable fluorescent dye. The hybridized slides are analyzed, and the abundance of protein-antibody in the test sample relative to that of the reference sample is assayed. cognate antibodies. Such an assay system has been validated by many groups $(10,22,23,34)$. Although such antigen arrays promise detection of interactions between antigen-antibody pairs, initial concerns in designing such an approach involved issues of sensitivity and specificity. With the aim of addressing these concerns, Haab et al. (10) used 115 antigen-antibody pairs for validating their antigen arrays. Their study used six different mixtures of antibody, each labeled with $\mathrm{Cy}^{\mathrm{TM}} 5$ dye (Amersham Biosciences, Piscataway, NJ, USA) and compared against a fixed concentration of Cy3-labeled reference mixture. The color of the antigen spots on the array varied concordantly with changes in the concentration of their binding partners in the probe mixture. The antigen array could detect antibodies at concentrations as low as $100 \mathrm{pg} / \mathrm{mL}$. This sensitivity was maintained even in the presence of 10- to 100-fold excess of fetal calf serum, indicating the feasibility of using this format to study changes in protein expression in complex solutions. Similarly, the antigen array reported by our group (34) comprised different concentrations of rabbit immunoglobulin (Ig) $G$ ranging from 1.6-50 pg. Such a chip, when probed with picogram quantities of anti-rabbit $\operatorname{IgG}$ had a linear range of detection across the concentrations tested, with a sensitivity of $6.25 \mathrm{pg}$ of spotted antigen. This antigen array was also highly specific when incubation mixtures contained a large excess of nonspecific proteins ( $1 \%$ bovine serum albumin [BSA]). Such specificity was maintained even when the chip contained 96 distinct elements. This indicated the ability to use an antigen chip to assess changes in protein-antibody levels in a complex biological milieu (34). In a similar approach, MacBeath and Schreiber demonstrated that the binding of spotted FKBP12-rapamycin binding (FRB) domain of FKBP-rapamycin associated protein (FRAP) to labeled human immunophilin FKBP12 occurred only in presence of rapamycin (22).

One of the major factors governing the issue of sensitivity of antigen arrays is the matrix used to immobilize the antigens. Thus, it becomes necessary to select the appropriate surface for spotting and binding antigens. There are a number of substrates available, many of which are based on modifications of a glass surface. These include poly-L-lysine-coated $(10,23,34)$, aldehyde-coated $(22,23,34)$, and nitrocellulose-coated surfaces $(14,23,27,34)$. Although aldehyde-coated slides promise to offer greater sensitivity compared to poly-L-lysine-coated matrices, presumably better sensitivity and lower signal-to-noise ratio have been obtained with nitrocellulose-coated slides (23). As an example, Figure 2 shows one of our antigen arrays using nitrocellulose slides. The array comprised recombinant heat-shock proteins (HSPs), transferrin, yeast cytosine deaminase (YCD), recombinant mitogen-activated protein (MAP)-kinase (ERK1), interferons (IFNs) ( $\alpha, \beta$ and $\gamma$ ), and BSA. Such an array showed high specificity and low background when probed with a cocktail of antibodies against HSPs (Figure 2). Although nitrocellulose slides offer a high signal-to-noise ratio and, hence, better sensitivity, a concern while using such matrices is the inability to direct the orientation of spotted proteins (23). One way to overcome this, is by using site-specific immobilization of proteins to substrates. This was demonstrated by Zhu et al., who used nickel-nitrilotriacetic acid (Ni-NTA)-derivatized slides to bind about 5800 His-tagged yeast open reading frames (38). In another approach, directional binding was achieved by site-specific bi- 
otinylation of proteins followed by their immobilization to avidin-coated glass slides (20). Both approaches were able to maintain functional activity and, presumably, orientation of a large percentage of spotted proteins.

Innovations that implement the antigen-protein microarray approach have resulted in development of a number of novel applications. These include high-throughput detection of enzyme-substrate interactions (22), identification of novel protein interacting domains (7), characterization of small molecule interactions with proteins (22), and detection of protein levels and/or activities in pure populations of tumor cells (27). One such innovative application of antigen arrays involved the investigation of interacting domains for various biomolecules involved in signaling pathways (7). The array consisted of recombinant proteins containing various interaction motifs on their surface. This "protein motif" biochip was validated using fluorescent streptavidin-tagged peptides that interact with each of these modules. Importantly, using this system, interacting domains for the signaling molecule Sam68 and core small nuclear ribonucleoprotein (snRNP) protein $\mathrm{SmB}$ could be detected (7). Since protein-protein interactions play a central role in the development and progression of cancer, it would be important to study such interacting modules in tumor samples using these biochips. Such studies may also help in designing molecules that inhibit these interactions and potentially interfere with the development and/or progression of cancer.

A further advance over the conventional antigen chip is the concept of the reverse phase antigen array, wherein a total cellular lysate is spotted (27). These can be probed either with specific antibodies, patient sera, or antigens. The lysates to be spotted are reduced and denatured to enable the probe to have access to all its epitopes. Levels of expression of various proteins across different samples are normalized against levels of a housekeeping protein such as actin. The validation of reverse phase array was carried out by spotting different amounts of recombinant prostate-specific antigen (PSA). The sensitivity of detection was as low as $10^{-21} \mathrm{~mol}$ of spotted antigen (27). Furthermore, Paweletz et al. combined reverse phase microarray with laser capture microdissection to isolate specific benign and malignant prostate epithelial cells to study changes in expression of proteins regulating prosurvival pathways (27). They were able to detect changes in levels of specific proteins as well as alterations in phosphorylation status. Their work shows a strong positive correlation between phosphorylation of Akt and cancer progression, which is augmented by activation of the other prosurvival pathway genes (27). This example illustrates the power of biochip technology, enabling comparison of multiple protein expression alterations across many samples. In our experience, we used a similar approach to examine levels of candidate cancer biomarkers in various prostate cell lines. The relative abundance of tumor antigens was determined as the ratio of relative abundance with respect to an internal standard. Such a normalization enabled comparison across different protein microarray experiments. As an example, Figure 3A shows a biochip containing denatured proteins from various cancer cell lines probed for expression levels of a novel tumor antigen discovered through our work (and designated UM2). Total proteins from six different cancer cell lines were spotted on nitrocellulose-coated slides and probed with a solution containing anti-UM2 (rab- bit polyclonal) and anti-actin (mouse monoclonal) antibodies. Species-specific secondary antibodies conjugated to $\mathrm{Cy} 3$ and Cy5 flourochromes, respectively, were used for detection. The relative abundance of the tumor antigen across the various cell lines was represented as the ratio of average median intensity of UM2 spots to the ratio of average median intensity of the actin spots. A gradation in the color of the spots from green to red was indicative of the relative abundance of UM2 levels across various cell lines. Graphical representation of the data showed highest levels of UM2 in the prostate cancer (PCA) cell line DUCAP $(1.6 \pm 0.2)(19)$ and lowest levels in the normal transformed prostate cell line RWPE $(0.16 \pm 0.02)$ (Figure 3B). Western blot analysis of these cell lines for UM2 showed concordance with the protein array data (Figure 3C). Here, the use of a 2-color system allows one to make direct comparisons between two samples and, at the same time, compensates for variability due to spotting and efficiency of labeling. In a similar approach,

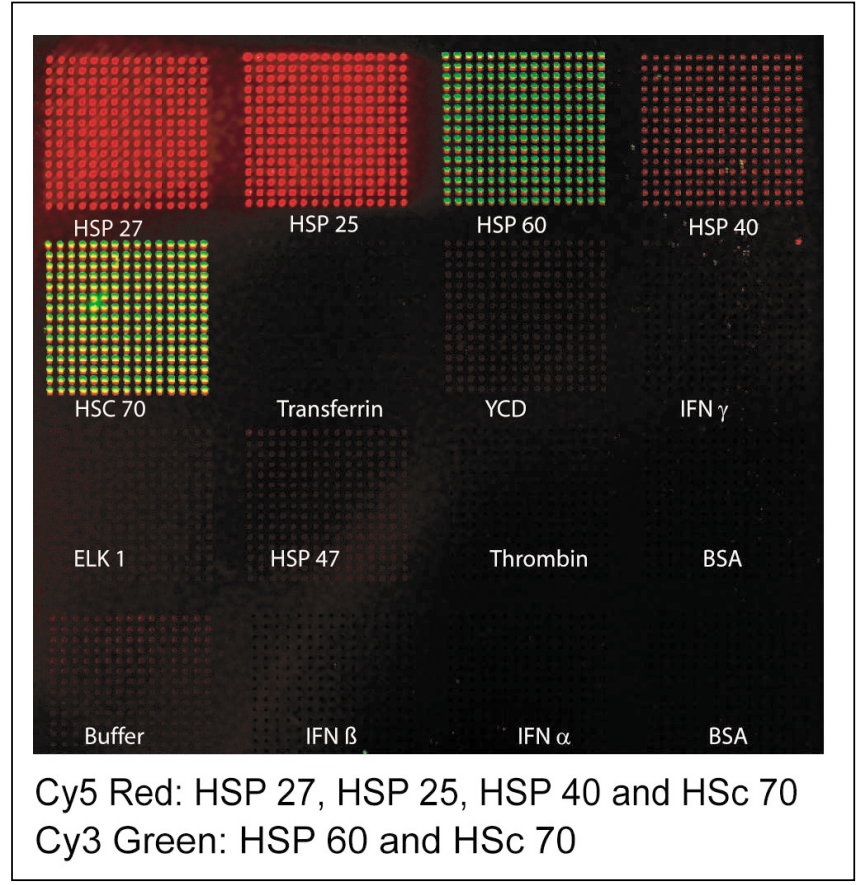

Figure 2. Detection of antigens on a protein microarray using labeled antibodies as probes. Various recombinant proteins, namely MAP kinase $(10 \mathrm{mg} /$ $\mathrm{mL}), \mathrm{YCD}(1.4 \mathrm{mg} / \mathrm{mL})$, heat-shock cognate (HSC) $70(1.25 \mathrm{mg} / \mathrm{mL})$, HSP 25, HSP 27, and transferrin (each $1 \mathrm{mg} / \mathrm{mL}$ ), HSP 40 and HSP 60 (each 0.7 $\mathrm{mg} / \mathrm{mL})$, HSP $47(0.6 \mathrm{mg} / \mathrm{mL})$, and IFN- $\alpha, \beta$ and $\gamma($ each $0.5 \mathrm{mg} / \mathrm{mL})$ and BSA $(1 \mathrm{mg} / \mathrm{mL})$ were spotted in replicates onto nitrocellulose-coated glass slides. After blocking the nonspecific sites, the slide containing the microarray was incubated with a primary antibody mixture containing rabbit polyclonal antibodies against HSP 27, HSP 25, HSC 70 (each diluted 1:100), and HSP 40 (1:300), and mouse monoclonal antibodies against HSP 60 (1:250) and HSC $70(1: 200)$. This was followed by incubation with a mixture of anti-rabbit antibody $(1: 100)$ labeled with $\mathrm{Cy} 5$ dye and anti-mouse antibody $(1: 100)$ labeled with Cy3 dye. The slide was scanned using a microarray scanner (Axon Instruments, Union City, CA, USA) and data was analyzed using GenePix ${ }^{\circledR}$ software. The pattern of immunoreactivity was concordant with the presence and/or absence of cognate antibody in the probe mixture. Thus spots corresponding to HSP 27, HSP 25, and HSP 40 appeared red, while spots corresponding to HSP 60 appeared green in color. Since antibodies to HSC 70 were present in both the channels, these spots appeared yellow on the array. Spots corresponding to other recombinant antigens or BSA did not show any immunoreactivity. 


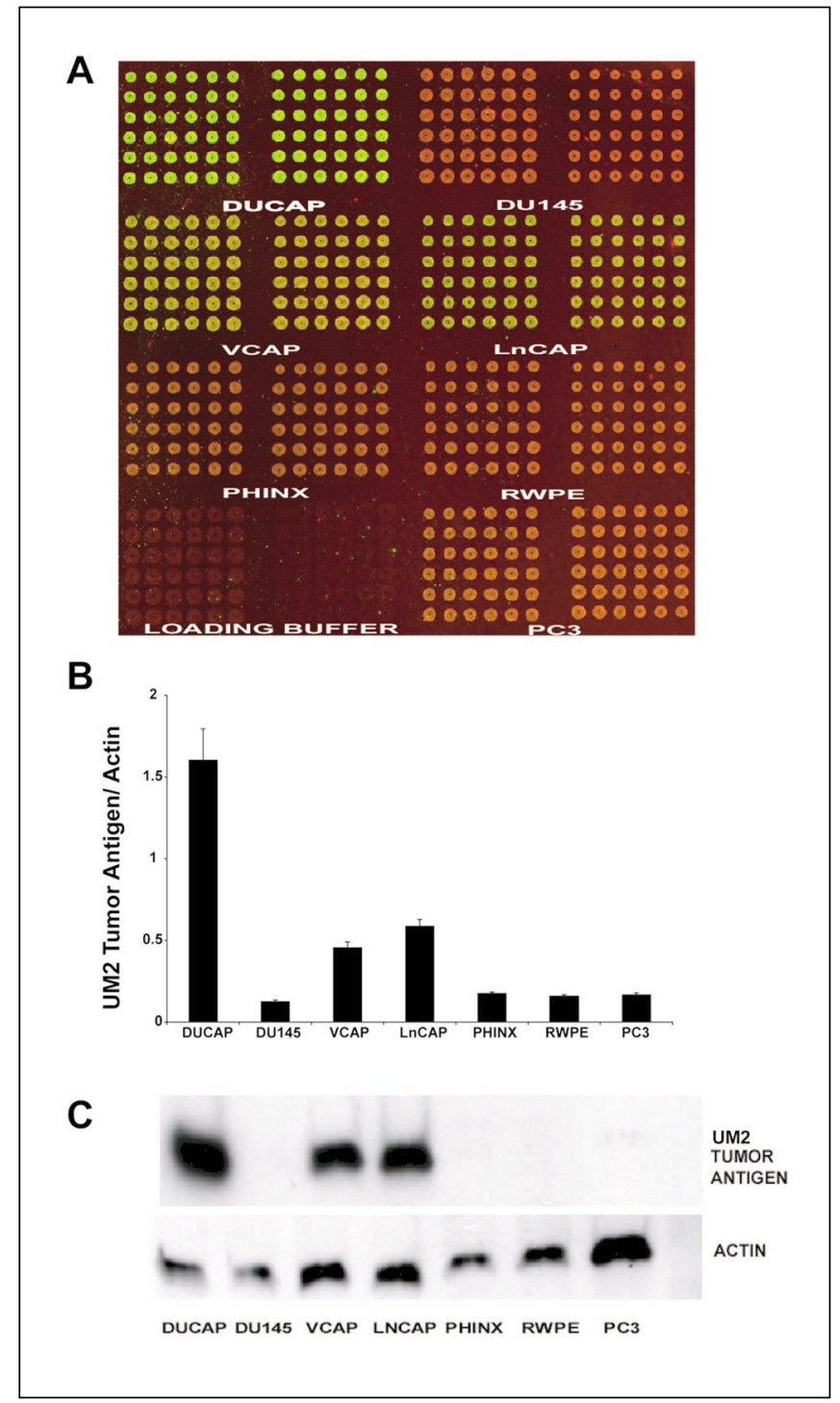

Figure 3. Reverse-phase protein microarray used to detect tumor antigens in cancer cell lysates. (A) Cell lysates from six PCA cell lines, namely DUCAP, DU145, VCAP, LnCAP, RWPE, and PC3, and human embryonic kidney cell line PHOENIX (each $1 \mathrm{mg} / \mathrm{mL}$ ) were lysed by boiling in Laemmli buffer (17) for 2 hours at $70^{\circ} \mathrm{C}$ followed by final denaturation at $100^{\circ} \mathrm{C}$ for 10 minutes. The denatured cell lysates were then arrayed onto nitrocellulose slides using a GMS $417^{\mathrm{TM}}$ arrayer (Affymetrix, Santa Clara, CA, USA). The microarray consisted of 72 spots for each cell type. Spots corresponding to the buffer served as negative controls. The slides containing the arrayed lysates were first probed with polyclonal antibodies against UM2 tumor antigen and monoclonal antibody against actin (each diluted 1:250). This was followed by incubation with a mixture containing anti-mouse antibodies labeled with $\mathrm{Cy} 5$ and anti-rabbit antibodies labeled with Cy3 (each diluted 1:250). Immunoreactivity was visualized by scanning the slides using a confocal laser scanner. The relative abundance of the tumor antigen across the various cell lines was represented as the ratio of average median intensity of UM2 spots to the ratio of average median intensity of the actin spots. A gradation in the color of the spots from green to red was indicative of the relative abundance of the UM2 levels across various cell lines. (B) Graphical representation of the data showed highest levels of UM2 in the prostate cancer cell line DUCAP $(1.6 \pm 0.2)$ followed by LnCAP $(0.6 \pm 0.04)$ and VCAP $(0.5 \pm$ $0.03)$. Lowest amounts of the tumor antigen were found in normal transformed prostate cell line RWPE $(0.16 \pm 0.009)$. (C) Immunoblot analysis of PCA cell lines with antibodies against UM2 tumor antigen and actin showed complete concordance with protein microarray. Highest levels of tumor antigen were found in DUCAP followed by LnCAP and VCAP, respectively.
Madoz-Gurpide et al. spotted fractionated proteins from lung adenocarcinoma cells and probed with antibodies directed against specific antigens (23). Such a system could facilitate the use of an entire repertoire of cellular proteins as probes to identify interacting partners and potential autoantibodies.

Reverse phase technology adds a new dimension to the existing high-throughput formats. The power of this system is the ability to analyze a large number of clinical samples (in minute quantities) for differential protein expression. In addition this system could also be used to look for antigen biomarkers in patient sera for various disease states, antibody response in individuals undergoing therapeutic regimens, screening expression libraries for protein-protein interactions, and screening hybridomas.

Among the many applications of antigen arrays, the one with the most potential for clinical utility is the ability to look for humoral response to various extrinsic proteins or tumor markers in sera. In one such approach, biochips spotted with various microbial antigens were used to detect specific antibody responses in serum of individuals with various microbial infections (25). The results obtained with such a miniaturized ELISA system had an overall concordance of greater than $80 \%$ with conventional ELISA, in the classification of the sera and offered the ability to detect as low as $0.5 \mathrm{pg}$ of serum IgG or IgM (25). This approach was extended by Robinson et al. to study the humoral response in autoimmune diseases (29). In their approach, the authors used an 1152 feature array containing 36 recombinant proteins and 154 overlapping and immunodominant peptides to study antibody response in 50 serum samples from individuals with different autoimmune disorders (29). They were able to identify specific antibody responses for each of the eight autoimmune conditions studied. The authors claim this assay system to be 4- to 8-fold more sensitive than a conventional ELISA in detecting autoantibodies to various self-antigens (29).

One of the clinically important applications of such an assay system would be in the study of humoral response to various tumor markers. Initial studies using conventional techniques like immunoblot analysis (18) and ELISA (24) have shown the existence of such a phenomena in sera from cancer patients. The above assay system would offer the power to perform population screening for such antibody repertoires. In our laboratory, we applied antigen arrays to look for humoral response in cancer patients. Our approach was driven by the prior knowledge that the humoral response would be generated against small peptide epitopes presented by major histocompatibility complex (MHC) antigens. Hence, we used denatured recombinant proteins as baits on a biochip (Figure 4). These antigen chips were probed with sera from patients with biopsy-proven prostate cancer and healthy controls. Human Igs were detected using secondary antibodies conjugated to flourochromes (Cy5 or $\mathrm{Cy} 3)$. Comparison across slides was achieved by using various internal controls, which included antibodies to human serum and Cy5labeled streptavidin. As an example, Figure 4 shows detection of the humoral response in serum from prostate cancer patients and healthy control individuals against selected cancer biomarkers. Interestingly, specific antibody response against UM2 biomarker was found in the sera of prostate cancer patients (Figure 4). Immune response to UM2 was not appreciably seen in the sera of normal healthy individuals. This was further validated by immunoblot analysis (Figure 4B). Such screens for tumor-spe- 
cific autoantibodies could facilitate early diagnosis of cancer, monitor its progression, and predict its recurrence. Also, such an assay system could be used to study the immune response elicited by vaccines and other therapeutic agents.

Analogous to DNA and protein biochips, Kononen et al. (15) described a method for evaluating tumor tissues in large numbers on a single glass slide. Broadly defined, antigen microarrays would also include immunohistochemical analysis of tissue microarrays. These high-density tissue microarrays allow for the analysis of up to 1000 tissue samples on a single slide. The slides that contain tissue sections prepared by hematoxylin and eosin (H\&E) staining, followed by immunohistochemical staining can be evaluated by routine light microscopy. Various groups including our own, have established the use of tissue microarrays for high-throughput immunohistochemistry (28), in situ hybridization (32), or fluorescence in situ hybridization (FISH) $(2,8,9)$. Thus, candidate cancer biomarkers, identified by high-throughput gene or protein expression methodologies, can be evaluated at the immunohistochemical level over a large number of tumor specimens.

\section{ANTIBODY MICROARRAYS}

The conventional methods relying on use of antibodies to probe protein expression changes include immunoblot analysis, ELISA, and RIA. These assay systems fail in their ability to simultaneously detect the array of protein expression changes occurring in a cell at any given time. Thus, a system combining the sensitivity of these techniques with the robustness of DNA microarrays would be useful for looking at large-scale changes in protein levels. Since development, progression and invasiveness of cancer are governed by large-scale changes in protein levels or activities, and thus, development of antibody microarrays would help in early detection of these events.

The concept underlying the generation of antibody arrays is shown in Figure 5. This biochip consists of spotted antibodies, which are probed with labeled purified antigens or complex solutions like cell lysates or serum. As in case of antigen array development, the major considerations here include the orientation of the antibody on the chip, sensitivity of detection, and specificity of interaction. The first caveat has been addressed by inducing directional binding of the antibody on protein G-coated slides and metal-coated matrices (38). The problem of sensitivity and specificity has been addressed by many groups $(10,14)$ including ours (34). In their antibody arrays, Haab et al. used 115 distinct antibodies and showed specific interaction with cognate antigens, with a sensitivity of $1 \mathrm{ng} / \mathrm{mL}$ (10). Further, to test its applicability in detecting changes in protein levels in complex milieu like cell lysates or sera, the authors labeled the antigens in the presence of a large excess of nonspecific proteins. Their data suggests that the limit of detection decreases proportionately with increase in the total protein content of the probe solution. This is illustrated by an 100-fold decrease in sensitivity of detection of FLAG ${ }^{\circledR}$-tagged bacterial alkaline phosphatase when the amount of total protein in the mixture was increased by 10 -fold (10). Similarly, the antibody array developed by our group could detect antigens to a concentration as low as $5 \mathrm{ng} / \mathrm{mL}$ in the background of a large excess of nonspecific protein (34). Although the above examples illustrate the ability of antibody arrays to detect antigens in the presence of large amounts of nonspecific proteins, the real power of this system depends on its ability to look for alterations in protein levels in cell lysates or serum samples.

Use of antibody arrays to monitor protein expression in cell lysates has been reported by different groups including ours $(14,34)$. In our study, we used a 1920 element antibody chip consisting of 141 distinct antibodies to profile alterations in protein expression in colon cancer cell lysates treated with ionizing radiation (34). Fluorescently labeled cell lysates were used to detect alterations in levels of proteins in response to radiation. As an example, an antibody array showing radiation-induced alterations in levels of proteins, such as DNA fragmentation factor 40 (DFF40)
A

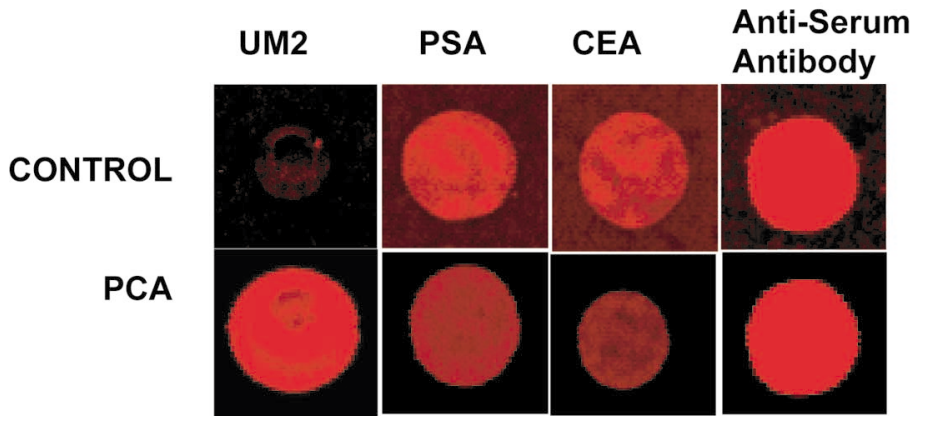

B

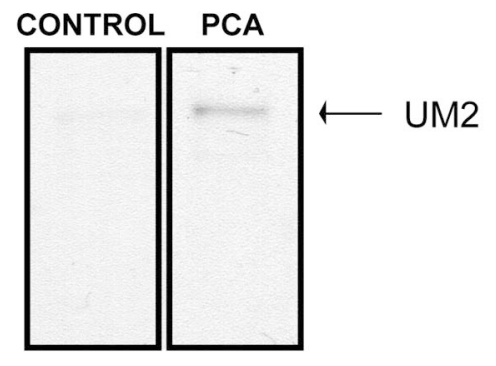

Figure 4. Reverse-phase protein microarray of recombinant tumor antigens to study humoral response in PCA serum. (A) Recombinant antigens such as tumor antigen UM2, PSA, and CEA (each $100 \mu \mathrm{g} / \mathrm{mL}$ ) were denatured and spotted onto nitrocellulose-coated slides. Antibody to total human serum (diluted 1:100) was used as an internal standard to compare across hybridizations. The slides were probed with serum (1:50) from either a normal individual (control) or PCA patient. Specific humoral response was seen against UM2 antigen in serum of only PCA patient. However, humoral response to PSA and CEA were seen in sera from both normal individual and PCA patient. Antibodies to total serum proteins reacted equally with serum from both normal individual and cancer patient. (B) Immunoblot analysis for UM2 probed with PCA serum or control (control). Specific band corresponding to UM2 was obtained with serum from prostate cancer patients. No bands were seen in blots probed with normal serum. 


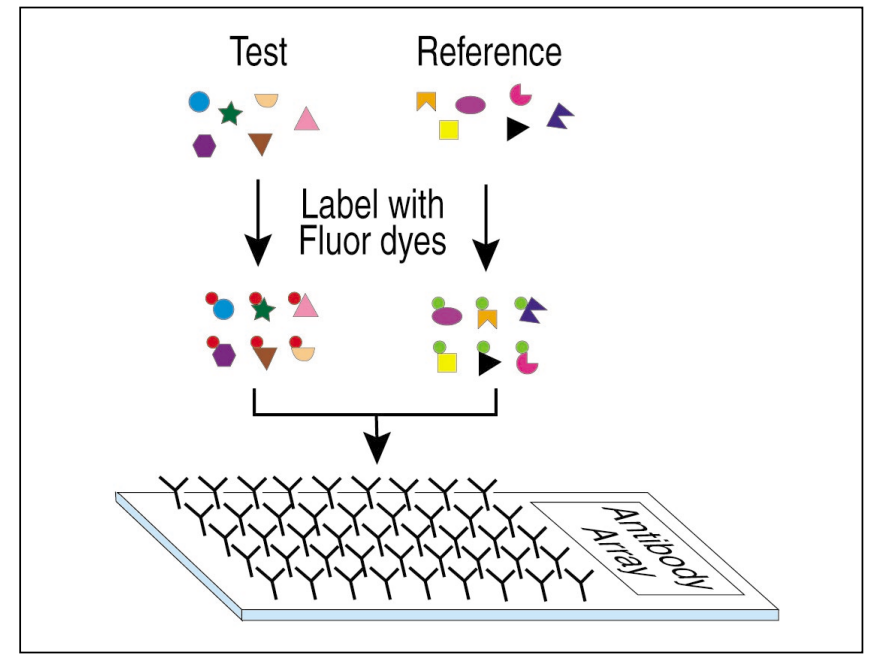

Figure 5. Schematic representation of the antibody microarray system used to monitor changes in protein expression in cancer cells. Proteins were extracted from reference or test samples and labeled with either Cy5- or Cy3-N-hydroxysuccinimide (NHS) esters. The labeled mixture was incubated with the microarrays. The slides were scanned using a confocal laser scanner, and the data was analyzed using GenePix software. Proteins were categorized as up-regulated, down-regulated, or unchanged, depending on their normalized average median of ratios in the test sample compared against the reference sample. and carcinoembryonic antigen (CEA), is shown in Figure 6. Importantly, our study brought out a number of novel associations between radiation and various apoptotic regulators like uncoupling protein 2 (UCP2), DFF40 and its inhibitor (DFF45), and FLICE-like inhibitory protein (FLIP), to name a few. As in the case of DNA microarrays, the use of a 2-color approach enabled a direct comparison to be made between treated and untreated samples (34). Similarly, using an antibody chip comprising 368 antibodies and microdissected populations of cancer cells, Knezevic et al. showed cancer-specific alterations in levels of various proteins using biotin-based signal amplification (14). Their study identified specific alterations in protein levels in defined cell types within the tumor that were correlated to tumor progression. This highlights the power of antibody arrays to study large-scale changes in protein expression using very small quantity of samples. An additional application of antibody arrays in the field of cancer research would be in the diagnosis and classification of tumors based on expression patterns of marker molecules. The conventional techniques, such as flow cytometry, have the inherent drawbacks of being labor-intensive and have the ability to analyze only a few biomarkers at a time (5). Also, in many cases, immunophenotypic data for a limited set of markers makes it difficult to define the tumor subtype (13) and, in most instances, necessitates the requirement for screening the expression of 50-100 


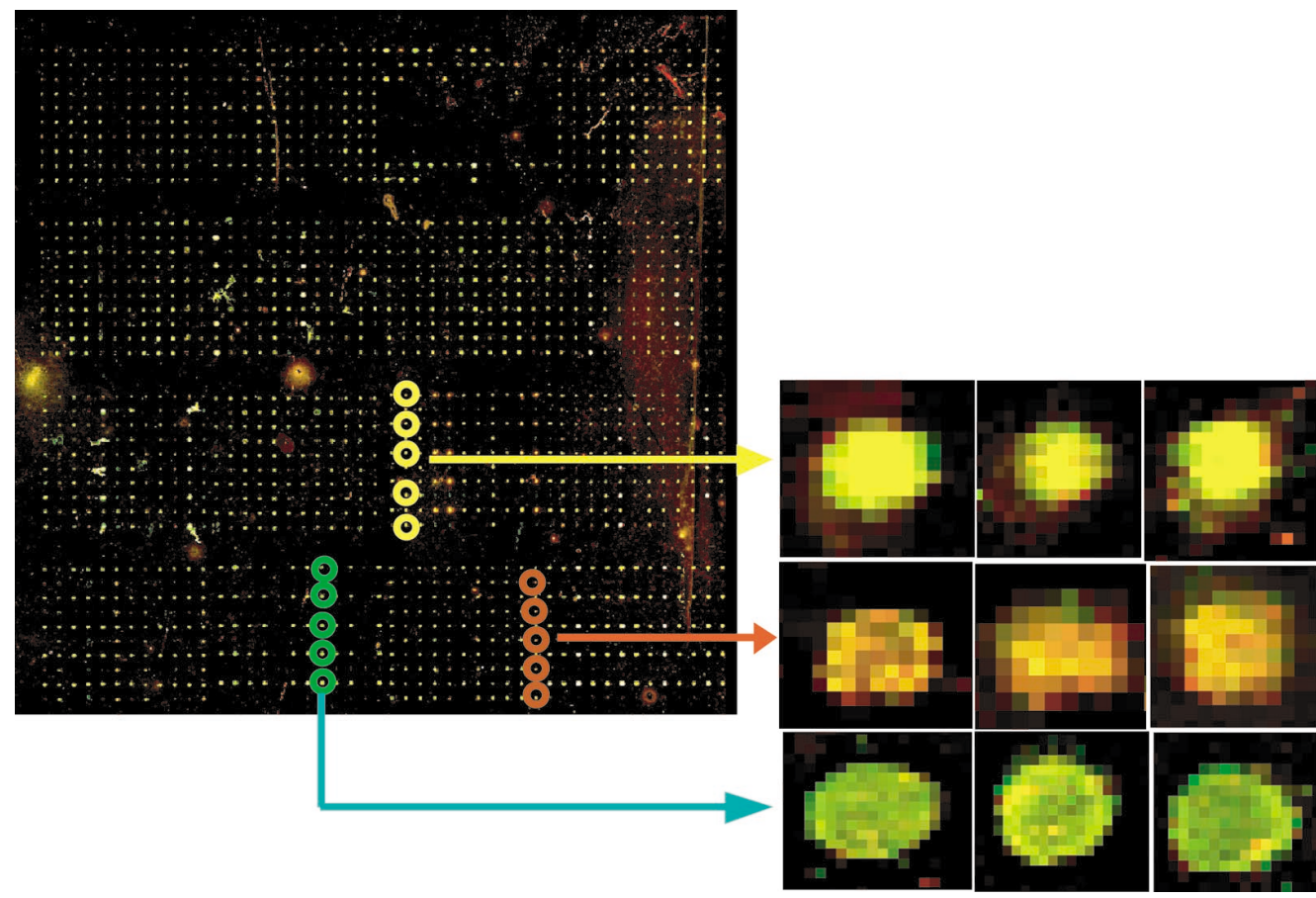

FADD

CEA

DFF40

Figure 6. Antibody array used to study protein expression in radiation-treated LoVo colon carcinoma cells. LoVo cells were irradiated with 4 Gy radiation and cultured for 4 hours at $37^{\circ} \mathrm{C}, 5 \% \mathrm{CO}_{2}$. One hundred micrograms of total proteins from radiation-treated LoVo cells and untreated controls were labeled separately with $\mathrm{Cy} 3$ and Cy5 dyes, respectively, and hybridized simultaneously on a 1920-element antibody array consisting of 146 distinct antibodies. There was a significant ( $p<$ $0.0001)$ increase in levels of DFF40 (green spots) upon radiation treatment. Interestingly, radiation treatment resulted in significant reduction $(P<0.0001)$ in levels of CEA (red spots). However, the levels of FADD were equal in both the lysates (yellow spots).

antigens (11). To address these requirements, an innovative approach combining the power of flow cytometry and antibody microarrays was used by Belov et al. to immunophenotype various classes of leukemia based on their expression pattern of cell surface cluster differentiation antigens (CD antigens) (5). Using antibodies against various $\mathrm{CD}$ antigens, they were able to determine expression levels of about $50 \mathrm{CD}$ antigens specific to leukocytes and leukemic cells (5). Importantly, this approach enabled the classification of closely related phenotypically distinct cancers.

An additional application of antibody arrays of clinical importance is in the field of cytokine research. Cytokines are known to interact with each other and in combination to elicit biological responses that differ markedly from the ones elicited by them in isolation (30). Although techniques like ELISA enable documentation of such responses, they have drawbacks in that they require large sample volumes for analysis (30). In this context, a multiplexed highly sensitive antibody microarray system combining the power of sandwich ELISA and rolling circle amplification (31) was developed for high-throughput detection of cytokines in biological samples (30). In their chemokine array, Schweitzer et al. measured 75 chemokines in various biological samples with high specificity, femtomolar sensitivity, 3-fold log dynamic range and very low amounts of sample (30). Such high sensitivity was achieved using rolling circle amplification (31). Comparison across chips was made possible using internal standards like labeled BSA or biotin-conjugated IgG (30). The availability of multiple antibodies, both monoclonal and polyclonal, for a large number of proteins make it possible to apply microarray-based
ELISAs to study protein-protein interactions, regulators of signal transduction cascades, and drug-target interactions.

\section{CONCLUDING REMARKS}

Development of high-throughput proteomics has led to a renaissance in approaches to look at protein expression and/or function in cancer cells, especially since proteins are the final "common denominator" in the development, progression, and invasion of tumors. Future efforts to develop a whole proteome chip would help in the early diagnosis of cancer, monitoring cancer progression, and designing rational therapies to target this disease. Challenges ahead include increasing the specificity and sensitivity of assay systems to enable monitoring microscopic changes in protein expression in cells.

\section{ACKNOWLEDGMENTS}

The authors wish to thank Dr. Bharathi Laxman for experimental help, and Drs. Chandan Kumar-Sinha and Ira Maine for their comments during preparation of the review. This work is supported by grants from the Mary Kay Ash Foundation, American Cancer Society (RSG 103013), the National Institutes of Health (ROI CA 97063), and National Cancer Institute (NCI) Specialized Program of Research Excellence (SPORE) in Prostate Cancer (NCI P50CA69568). A.M.C. is a Pew Biomedical Scholar. 


\section{REFERENCES}

1.Alizadeh, A.A., M.B. Eisen, R.E. Davis, C. Ma, I.S. Lossos, A. Rosenwald, J.C. Boldrick, H. Sabet, et al. 2000. Distinct types of diffuse large B-cell lymphoma identified by gene expression profiling [see comments]. Nature 403:503-511.

2.Andersen, C.L., G. Hostetter, A. Grigoryan, G. Sauter, and A. Kallioniemi. 2001. Improved procedure for fluorescence in situ hybridization on tissue microarrays. Cytometry 45:83-86.

3.Armstrong, S.A., J.E. Staunton, L.B. Silverman, R. Pieters, M.L. den Boer, M.D. Minden, S.E. Sallan, E.S. Lander, T.R. Golub, and S.J. Korsmeyer. 2002. MLL translocations specify a distinct gene expression profile that distinguishes a unique leukemia. Nat. Genet. 30:41-47.

4.Beer, D.G., S.L. Kardia, C.C. Huang, T.J. Giordano, A.M. Levin, D.E. Misek, L. Lin, G. Chen, et al. 2002. Gene-expression profiles predict survival of patients with lung adenocarcinoma. Nat. Med. 8:816-824.

5.Belov, L., O. de la Vega, C.G. dos Remedios, S.P. Mulligan, and R.I. Christopherson. 2001. Immunophenotyping of leukemias using a cluster of differentiation antibody microarray. Cancer Res. 61:4483-4489.

6.Dhanasekaran, S.M., T.R. Barrette, D. Ghosh, R. Shah, S. Varambally, K. Kurachi, K.J. Pienta, M.A. Rubin, and A.M. Chinnaiyan. 2001. Delineation of prognostic biomarkers in prostate cancer. Nature 412:822-826.

7.Espejo, A., J. Cote, A. Bednarek, S. Richard, and M.T. Bedford. 2002. A protein-domain microarray identifies novel protein-protein interactions. Biochem. J. 367:697-702.

8.Fuller, C.E. and A. Perry. 2002. Fluorescence in situ hybridization (FISH) in diagnostic and investigative neuropathology. Brain Pathol. 12:67-86.

9.Gancberg, D., A. Di Leo, G. Rouas, T. Jarvinen, A. Verhest, J. Isola, M.J. Piccart, and D. Larsimont. 2002. Reliability of the tissue microarray based FISH for evaluation of the HER-2 oncogene in breast carcinoma. J. Clin. Pathol. 55:315-317.

10.Haab, B.B., M.J. Dunham, and P.O. Brown. 2001. Protein microarrays for highly parallel detection and quantitation of specific proteins and antibodies in complex solutions. Genome Biol. 2:RESEARCH0004.

11.Harris, N.L., E.S. Jaffe, J. Diebold, G. Flandrin, H.K. Muller-Hermelink, J. Vardiman, T.A. Lister, and C.D. Bloomfield. 1999. World Health Organization classification of neoplastic diseases of the hematopoietic and lymphoid tissues: report of the Clinical Advisory Committee meeting-Airlie House, Virginia, November 1997. J. Clin. Oncol. 17:3835-3849.

12.Hirsch, M.S., B. Conway, R.T. D’Aquila, V.A. Johnson, F. Brun-Vezinet, B. Clotet, L.M. Demeter, S.M. Hammer, et al. 1998. Antiretroviral drug resistance testing in adults with HIV infection: implications for clinical management. International AIDS Society_USA Panel. JAMA 279:1984-1991.

13.Jennings, C.D. and K.A. Foon. 1997. Recent advances in flow cytometry: application to the diagnosis of hematologic malignancy. Blood 90:28632892.

14.Knezevic, V., C. Leethanakul, V.E. Bichsel, J.M. Worth, V.V. Prabhu, J.S. Gutkind, L.A. Liotta, P.J. Munson, E.F. Petricoin, III, and D.B. Krizman. 2001. Proteomic profiling of the cancer microenvironment by antibody arrays. Proteomics 1:1271-1278.

15.Kononen, J., L. Bubendorf, A. Kallioniemi, M. Barlund, P. Schraml, S. Leighton, J. Torhorst, M.J. Mihatsch, G. Sauter, and O.P. Kallioniemi. 1998. Tissue microarrays for high-throughput molecular profiling of tumor specimens. Nat. Med. 4:844-847.

16.Kumar-Sinha, C., S. Varambally, A. Sreekumar, and A.M. Chinnaiyan. 2002. Molecular cross-talk between the TRAIL and interferon signaling pathways. J. Biol. Chem. 277:575-585.

17.Laemmli, U.K. 1970. Cleavage of structural proteins during the assembly of the head of bacteriophage T4. Nature 227:680-685.

18.Le Naour, F., D.E. Misek, M.C. Krause, L. Deneux, T.J. Giordano, S. Scholl, and S.M. Hanash. 2001. Proteomics-based identification of RS/DJ1 as a novel circulating tumor antigen in breast cancer. Clin. Cancer. Res. 7:3328-3335.

19.Lee, Y.G., S. Korenchuk, J. Lehr, S. Whitney, R. Vessela, and K.J. Pienta. 2001. Establishment and characterization of a new human prostatic cancer cell line: DuCaP. In Vivo 15:157-162.

20.Lesaicherre, M.L., R.Y. Lue, G.Y. Chen, Q. Zhu, and S.Q. Yao. 2002. Intein-mediated biotinylation of proteins and its application in a protein microarray. J. Am. Chem. Soc. 124:8768-8769.

21.Lipshutz, R.J., S.P. Fodor, T.R. Gingeras, and D.J. Lockhart. 1999. High density synthetic oligonucleotide arrays. Nat. Genet. 21:20-24.

22.MacBeath, G. and S.L. Schreiber. 2000. Printing proteins as microarrays for high-throughput function determination. Science 289:1760-1763.

23.Madoz-Gurpide, J., H. Wang, D.E. Misek, F. Brichory, and S.M. Hanash. 2001. Protein based microarrays: a tool for probing the proteome of cancer cells and tissues. Proteomics 1:1279-1287.

24.McNeel, D.G., L.D. Nguyen, B.E. Storer, R. Vessella, P.H. Lange, and M.L. Disis. 2000. Antibody immunity to prostate cancer associated antigens can be detected in the serum of patients with prostate cancer. J. Urol. 164:1825-1829.

25.Mezzasoma, L., T. Bacarese-Hamilton, M. Di Cristina, R. Rossi, F. Bistoni, and A. Crisanti. 2002. Antigen microarrays for serodiagnosis of infectious diseases. Clin. Chem. 48:121-130.

26.Pandey, A. and M. Mann. 2000. Proteomics to study genes and genomes. Nature 405:837-846

27.Paweletz, C.P., L. Charboneau, V.E. Bichsel, N.L. Simone, T. Chen, J.W. Gillespie, M.R. Emmert-Buck, M.J. Roth, I.E. Petricoin, and L.A. Liotta. 2001. Reverse phase protein microarrays which capture disease progression show activation of pro-survival pathways at the cancer invasion front. Oncogene 20:1981-1989.

28.Perrone, E.E., C. Theoharis, N.R. Mucci, S. Hayasaka, J.M. Taylor, K.A Cooney, and M.A. Rubin. 2000. Tissue microarray assessment of prostate cancer tumor proliferation in African- American and white men. J. Natl. Cancer Inst. 92:937-939.

29.Robinson, W.H., C. DiGennaro, W. Hueber, B.B. Haab, M. Kamachi, E.J. Dean, S. Fournel, D. Fong, et al. 2002. Autoantigen microarrays for multiplex characterization of autoantibody responses. Nat. Med. 8:295-301.

30.Schweitzer, B., S. Roberts, B. Grimwade, W. Shao, M. Wang, Q. Fu, Q. Shu, I. Laroche, et al. 2002. Multiplexed protein profiling on microarrays by rolling-circle amplification. Nat. Biotechnol. 20:359-365.

31.Schweitzer, B., S. Wiltshire, J. Lambert, S. O'Malley, K. Kukanskis, Z. Zhu, S.F. Kingsmore, P.M. Lizardi, and D.C. Ward. 2000. Inaugural article: immunoassays with rolling circle DNA amplification: a versatile platform for ultrasensitive antigen detection. Proc. Natl. Acad. Sci. USA 97:1011310119.

32.Skacel, M., B. Skilton, J.D. Pettay, and R.R. Tubbs. 2002. Tissue microarrays: a powerful tool for high-throughput analysis of clinical specimens: a review of the method with validation data. Appl. Immunohistochem. Mol. Morphol. 10:1-6.

33.Slonim, D.K. 2001. Transcriptional profiling in cancer: the path to clinical pharmacogenomics. Pharmacogenomics 2:123-136.

34.Sreekumar, A., M.K. Nyati, S. Varambally, T.R. Barrette, D. Ghosh, T.S Lawrence, and A.M. Chinnaiyan. 2001. Profiling of cancer cells using protein microarrays: discovery of novel radiation-regulated proteins. Cancer Res. 61:7585-7593.

35.Stearns, V., H. Yamauchi, and D.F. Hayes. 1998. Circulating tumor markers in breast cancer: accepted utilities and novel prospects. Breast Cancer Res. Treat. 52:239-259.

36. Takahashi, M., D.R. Rhodes, K.A. Furge, H. Kanayama, S. Kagawa, B.B. Haab, and B.T. Teh. 2001. Gene expression profiling of clear cell renal cell carcinoma: gene identification and prognostic classification. Proc. Natl. Acad. Sci. USA 98:9754-9759.

37.Welsh, J.B., P.P. Zarrinkar, L.M. Sapinoso, S.G. Kern, C.A. Behling, B.J. Monk, D.J. Lockhart, R.A. Burger, and G.M. Hampton. 2001. Analysis of gene expression profiles in normal and neoplastic ovarian tissue samples identifies candidate molecular markers of epithelial ovarian cancer. Proc. Natl. Acad. Sci. USA 98:1176-1181.

38.Zhu, H., M. Bilgin, R. Bangham, D. Hall, A. Casamayor, P. Bertone, N. Lan, R. Jansen, et al. 2001. Global analysis of protein activities using proteome chips. Science 293:2101-2105.

\section{Address correspondence to:}

Dr. Arul M. Chinnaiyan

Department of Pathology

1301 Catherine Street, MSI Rm 4237

University of Michigan

Ann Arbor, MI 48109-0602, USA

e-mail:arul@umich.edu 